\title{
Experiencia de un Programa de Control de Asma Bronquial en Puerto Montt, Chile
}

\author{
MAURICIO RIQUELME O.*, RAÚL RIQUELME O.* y DORISA MARTÍNEZ R.**
}

\section{Report of a bronchial asthma control program in Puerto Montt, Chile}

Asthma is a disease with a variable clinical behaviour and usually insufficiently treated and managed. Methods: An Asthma Control Program was implemented in Puerto Montt, Chile, with the out patient participation of the primary medical care system. This Program classifies the asthmatic patients by using preestablished criteria of severity. We analyzed the evolution of 135 patients through six quarterly medical controls covering 18 months of follow up. Results: Patients diminished progressively and significantly their severity levels and their daily and nocturnal symptoms. Besides a significant increase of physical capacity $(p=0.001)$ and peak expiratory flow $(P E F)(p=0.0001)$ was observed. A series of severe asthmatic patients (12.5\%) was identified. This group concentrated most of the emergency consultations and hospitalizations. Conclusions: An organized management of asthmatic patients has allowed us to show objectively clinical improvements in these patients after entering to this program.

Key words: Bronchial asthma, control program, severity.

\section{Resumen}

El asma es una patología de comportamiento clínico variable y generalmente sub tratada. Material y métodos: En Puerto Montt se implementó un Programa de Control de Asma Bronquial integrado con el nivel primario de atención, que clasifica los pacientes usando criterios de gravedad. Se analizó la evolución de 135 asmáticos en 6 controles trimestrales cubriendo 18 meses de seguimiento. Resultados: Hubo disminución progresiva y significativa de los niveles de severidad, de síntomas diurnos y nocturnos, aumento de la capacidad de ejercicio $(p=0,001)$ y del flujo espiratorio máximo (PEF) (0,0001). Se identificó un subgrupo de asmáticos graves (12,5\%) responsables de la gran mayoría de las consultas en urgencia y de las hospitalizaciones. Conclusiones: El manejo organizado de los pacientes asmáticos permitió obtener ventajas clínicas objetivables.

Palabras claves: Asma bronquial, programa de control, gravedad.

\section{Introducción}

El asma bronquial es una enfermedad de prevalencia aún no bien conocida en Chile, que genera gran número de consultas y hospitalizaciones e incluye pacientes con características clínicas muy variables cuyas demandas de recursos no son homogéneas. El policlínico de Broncopulmonar (PB) del Hospital de Puerto Montt en forma histórica no tenía una clara coordinación con los niveles de atención primaria de salud, lo que determinaba una acumulación de pacientes leves o moderados que copaban y hacían insuficiente los horarios de los especialistas, retardando la atención de interconsultas o evaluación de pacientes complejos. Por esta razón nos pareció importante identificar subgrupos de asmáticos con presentaciones clínicas semejantes a fin de definir mejor su nivel de atención. La agrupación por criterios de

Policlínico de Broncopulmonar, Hospital de Puerto Montt, Chile.

* Neumólogo.

** Enfermera. 
gravedad cumple con estas premisas y llevar registros objetivos e informáticos permite evaluar el impacto clínico de las acciones de un programa de salud. El asma bronquial leve puede manejarse bien en la atención primaria, pero las formas moderadas y severas deben ser evaluadas por especialistas y eventualmente concentrarse en centros de mayor complejidad. Mostramos los resultados clínicos obtenidos con un programa de control de pacientes asmáticos en el Servicio de Salud Llanchipal (Llanquihue, Chiloé, Palena) clasificándolos según criterios de gravedad (leves, moderados y severos).

\section{Material y Métodos}

En el PB del Hospital de Puerto Montt, (hospital general de 400 camas), se implementó un Programa de Control de Asma bronquial basado en recomendaciones propuestas por la Sociedad Chilena de Enfermedades Respiratorias ${ }^{1}$ que luego se extendió progresiva y coordinadamente a la Atención Primaria y hospitales del área. En este Programa se ingresan aquellos pacientes que cumplen criterios diagnósticos de asma bronquial $^{2}$ practicándoseles una breve encuesta epidemiológica y aplicando un protocolo utilizado por el Instituto Nacional del Tórax previo a la puesta en marcha de las Salas ERA para control ambulatorio de crónicos (Tabla 1). Este permite obtener un puntaje de gravedad en cada consulta médica según frecuencia de síntomas diurnos, nocturnos, tolerancia al ejercicio (determinada según la dificultad para realizar labores habituales), frecuencia de crisis de asma, volumen espiratorio forzado en el primer segundo $\left(\mathrm{VEF}_{1}\right)$ o flujo espiratorio máximo $(\mathrm{PEF})$ y necesidades de tratamiento siendo clasificados en leves, moderados y severos ${ }^{1}$. Son controlados por médico cada 3 meses o según necesidad clínica, por enfermera en forma mensual y reciben periódicamente refuerzos educacionales sobre su patología, tratamiento y técnica inhalatoria. En aquellos pacientes residentes en Puerto Montt se define el lugar de control (PB o Consultorio Municipal). Aquellos que residen fuera de Puerto Montt son diagnosticados y controlados en su lugar de origen y sólo son enviados en interconsulta al PB si su médico tratante lo estima necesario. Se aumentó la cobertura de las espirometrías en el hospital y bajo supervisión del $\mathrm{PB}$, se agregaron dos espirómetros en los Consultorios y se incorporó un aparato portátil que rota periódicamente por establecimientos que no cuentan con dicho equipo. Además se implementó el uso rutinario de flujometría en cada control. La implementación terapéutica incluyó el uso de Salbutamol y Beclometasona o Budesonida inhalatoria según disponibilidad, uso rutinario de aerocámaras y prednisona oral en curas cortas en las crisis.

Se evalúa la situación clínica antes del ingreso al programa de 611 asmáticos y se presenta la evolución de 135 de ellos que se controlan en el PB del Hospital de Puerto Montt entre el $1^{\circ}$ de Diciembre de 1998 y 30 de Abril de 2004 y que completaron 6 controles médicos, analizando y comparando los datos obtenidos entre los distintos controles.

\section{Tabla 1. Evaluación de la gravedad en pacientes con asma bronquial}
Síntomas diurnos
a) Ausentes u ocasionales
b) $<2$ veces al mes
c) $>2$ veces al mes o semanal
d) Diarios
d) Diarios

Frecuencia asma nocturna
a) Ausentes u ocasionales
b) $<2$ veces al mes
c) $>2$ veces al mes o semanal
d) Todos los días

Tolerancia al ejercicio y asistencia al colegio/trabajo
a) Buena y no limitada
b) Disminuida, pero hace vida normal
c) Muy limitada

Ataques de asma
a) Ausentes o intermitentes (c/3 meses)
b) $<2$ veces a la semana Sin visita a Urgencia
c) $>2$ veces a la semana Visitas de Urgencia < 3 veces al año
d) Diaria y medicación idem
Visitas de Urgencia > 3 veces al año
e) Hospitalización por asma en 12 meses

\section{1}

$\mathrm{VEF}_{1}$ o PEF (\% teórico)
a) $>$ de $80 \%$
b) $50-79 \%$
c) $<50 \%$

Requerimientos de tratamiento
a) No necesita
b) $\beta 2$ Agonista aerosol
c) $\beta 2$ Agonista + esteroides aerosol $(<1.000 \mu \mathrm{g}) \quad 3$
d) $\beta 2$ Agonista + esteroides aerosol $(>1.000 \mu \mathrm{g})$ + esteroides orales

Puntaje total
Ocasional $=1-2$
Leve $=3-6$
Moderado $=7-13$
Severo $=14$ y más 
Análisis estadístico: Los resultados son expresados como media \pm DS. Para la comparación de las variables continuas se usó la prueba "t" de Student; las variables categóricas se compararon mediante la prueba $\chi^{2}$ y el test exacto de Fisher cuando fue necesario. Un valor de $\mathrm{p}<$ 0,05 se consideró estadísticamente significativo. Las variables obtenidas fueron almacenadas y analizadas en el programa estadístico SPSS para Windows 10.0 (SPSS, Chicago, IL, USA).

\section{Resultados}

En el periodo evaluado se ingresaron 611 pacientes, correspondiendo a asma ocasional el $5 \%$, leve $28,3 \%$, moderado $46,4 \%$ y severo 20,3\%. La situación clínica del año previo al ingreso al programa respecto de consultas en Urgencia, hospitalización una o más veces y el tipo de tratamiento que llevaban se exponen en la Tabla 2. A su ingreso referían haber tenido crisis obstructivas el $44,1 \%$, disnea el $88,1 \%$, sibilancias el $85,6 \%$, tos el $85,6 \%$, esputo el $68,6 \%$. Tenían síntomas desde hacía más de 2 años el 85,6\%, entre 6 meses y 2 años el 10,3\% y menos de 6 meses el 3,4\%. Los síntomas se describían como perennes en el $46,2 \%$ y de predominio en otoño-invierno en el $41 \%$ y primavera-verano en el 12,8\%. El 41,5\% recono- cía desencadenantes específicos en el asma, el $32,2 \%$ reaccionaba ante desencadenantes inespecíficos y un $29,7 \%$ no reconocía desencadenantes. El 9,3\% tenía rinitis concomitante. Destacaba la baja adherencia al uso constante de esteroides inhalados indicados además de emplear una técnica inhalatoria deficiente. No se consignó el uso de medicación empírica o inadecuada para el manejo de la patología.

El grado de severidad clínica de los pacientes varió según el lugar en que se controlaban, ya que los más graves se concentraron en el policlínico de especialidad. Las diferencias entre el PB y los consultorios municipales (CM) urbanos de Puerto Montt, Puerto Varas y Llanquihue se exponen en la Tabla 3.

En aquellos pacientes que se controlan en el PB hubo 135 casos que habían completado al menos 6 controles. El 72,6\% eran mujeres, edad $54,6 \pm 14,1$ años. Hubo cambios progresivos significativos entre el primer y el sexto control en la distribución de severidad tendiendo a formas más leves, observándose que el porcentaje de asmáticos severos disminuyó de $40,1 \%$ a $24 \%$. Disminuyeron los síntomas tanto diurnos como nocturnos, hubo aumento de la capacidad de ejercicio, del PEF y una disminución del puntaje de gravedad total del grupo de asmáticos (Tabla 4).

La frecuencia de hospitalizaciones, que en el

Tabla 2. Características de 611 asmáticos antes de ingresar al programa

\begin{tabular}{lccc}
\hline & $\begin{array}{c}\text { Leves / ocasionales } \\
\mathbf{2 0 4}(\mathbf{3 3 , 3 \% )}\end{array}$ & $\begin{array}{c}\text { Moderados } \\
\mathbf{2 8 4}(\mathbf{4 6 , 4 \%})\end{array}$ & $\begin{array}{c}\text { Graves } \\
\mathbf{1 2 3}(\mathbf{2 0 , 3 \% )}\end{array}$ \\
\hline Consultas en servicio de urgencia* & 4 & 51 & 69 \\
Hospitalización* & 0 & 15 & 32 \\
Sin tratamiento & 5 & 0 & 0 \\
Sólo $\beta 2$ agonista & 82 & 29 & 0 \\
$\beta 2+$ Beclometasona $<1.000 \mu \mathrm{g} /$ día & 114 & 227 & 40 \\
$\beta 2+$ Beclometasona $\geq 1.000 \mu \mathrm{g} /$ día & 3 & 28 & 83 \\
\hline
\end{tabular}

* 1 ó más veces en los 12 meses previo al ingreso

Tabla 3. Tipos de asma según criterios de gravedad y lugar de control

\begin{tabular}{lccccc}
\hline \multicolumn{2}{l}{ Tipo asma } & PB Puerto Montt & CM Puerto Montt & CM Puerto Varas & CM Llanquihue \\
\hline Leve & $(\%)$ & 17 & 51 & 38 & 72 \\
Moderado $(\%)$ & 45 & 47 & 52 & 26 \\
Grave & $(\%)$ & 38 & 2 & 10 & 2 \\
\hline
\end{tabular}

PB: Policlínico de Broncopulmonar. CM: Consultorio Municipal 
Tabla 4. Evolución clínica entre el $1^{\mathrm{er}}$ y $6^{\circ}$ control en 135 asmáticos

\begin{tabular}{lccc}
\hline & $\begin{array}{c}\text { Primer control } \\
\mathbf{n} / \text { \% }\end{array}$ & $\begin{array}{c}\text { Sexto control } \\
\mathbf{n} / \%\end{array}$ & p \\
\hline Síntomas leves & $28 / 20,7$ & $55 / 40,7$ & 0,019 \\
Sin crisis o molestias < 2 veces/semana & $79 / 58,5$ & $99 / 73,3$ & 0,001 \\
Sintomático todos los días & $75 / 55,6$ & $46 / 34,1$ & 0,010 \\
Sintomático todas las noches & $46 / 34,1$ & $29 / 21,5$ & 0,001 \\
Asintomático en las noches & $48 / 35,6$ & $77 / 57$ & 0,001 \\
Sin limitación para realizar ejercicio & $29 / 21,5$ & $47 / 34,8$ & 0,001 \\
Requirieron hospitalización por asma* & $17 / 12,6$ & $8 / 5,9$ & 0,063 \\
PEF L/min (media \pm DS) & $59 \pm 24$ & $61 \pm 23$ & 0,0001 \\
Puntaje de gravedad (media \pm DS) & $11,7 \pm 5,1$ & $10,1 \pm 5,3$ & 0,0001 \\
\hline
\end{tabular}

Síntomas leves: asintomático o $<2$ veces al mes.

* en los últimos 12 meses.

año previo al ingreso fue de $12,6 \%$, disminuyó progresivamente a $5,9 \%$, sin llegar a ser esta diferencia estadísticamente significativa $(\mathrm{p}=$ 0,063) (Figura 1) .

Logramos identificar un grupo de pacientes $(12,5 \%)$ de gran severidad, en los que pese a las medidas terapéuticas que incluían el cambio de Beclometasona por Budesonida, uso permanente de Salbutamol o Bromuro de Ipratropio asociado a Fenoterol, mejoramiento de la calidad de la aerocámara, controles frecuentes, refuerzos educativos al paciente y familiares, persisten con un perfil clínico diferente al compararlos con el resto de la población de asmáticos estudiados: casi todos persisten al $6^{\circ}$ control con asma nocturna ( $88 \%$ vs $12,3 \%)$, asma diurna persistente ( $94 \%$ vs $26,3 \%)$, deficiente tolerancia al ejercicio ( $82 \%$ vs $10,5 \%)$, consultan en el servicio de urgencia muy frecuentemente $(47 \%$ $v s 3,5 \%)$ y requieren más hospitalizaciones $(35 \%$ vs $1,8 \%)$.

\section{Comentario}

El presente estudio demuestra que un programa de control de pacientes asmáticos que integra un policlínico de especialidad con el nivel primario de atención, con buena comunicación con las emergentes salas ERA, usando criterios diagnósticos y terapias comunes, y agrupando según criterios de gravedad, permite disminuir la severidad del asma en un grupo de estos pacientes.

En el asma bronquial, el uso de recursos guarda relación directa con la gravedad. Los costos totales de un asma moderado pueden duplicar a

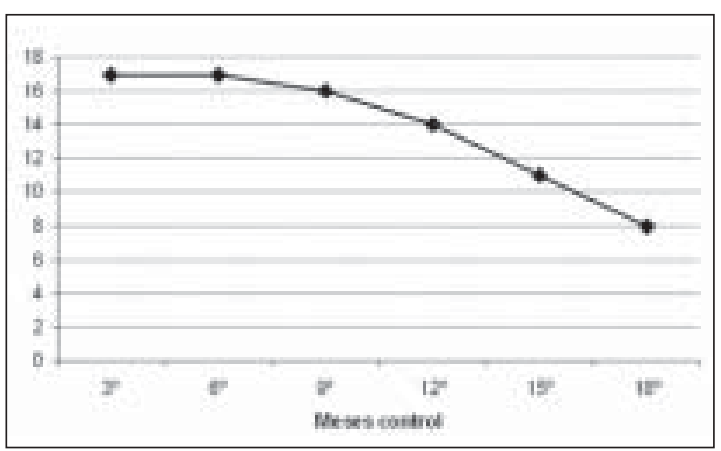

Figura 1. Número de hospitalizaciones en 6 controles sucesivos de 135 pacientes asmáticos.

los del asma leve y una forma grave, quintuplicarlo $^{7}$. Nuestra experiencia mostró que los pacientes más graves presentaron mayor número de consultas en Urgencia, hospitalizaciones y consumo de medicamentos, por lo cual deben controlarse en policlínicos de especialidad. El diagnóstico de una enfermedad crónica debe establecerse sobre bases seguras, razón por la que nos parece importante destacar los esfuerzos para facilitar el acceso a espirometrías estandarizadas y el uso rutinario de la flujometría como un índice objetivo en la evaluación de estos pacientes.

La clasificación del asma de acuerdo a su severidad tiene un carácter dinámico y facilita la indicación del tratamiento ${ }^{8}$. En nuestra serie hubo una disminución significativa de los niveles de severidad, de los síntomas diurnos y nocturnos, la frecuencia de las exacerbaciones y de las hospitalizaciones, tendencia que debería permanecer en el tiempo en la medida que se mantenga el control y tratamiento de los pacientes. 
Esto permitiría agilizar el flujo de pacientes entre los niveles de atención optimizando el uso de los policlínicos de especialidad. El control y disminución de la frecuencia de los pacientes más graves, que son los que utilizan más recursos permitirían lograr como ha sido señalado ${ }^{9}$, una reducción en los costos directos de la enfermedad (atención ambulatoria, de urgencia, hospitalizaciones, exámenes, etc) y también de los costos indirectos (pérdidas de días laborales, estudio, licencias médicas, etc). En nuestra realidad hemos comprobado que con pocos medicamentos y aplicando pautas educativas simples, se pueden lograr beneficios importantes ya que inversiones comparativamente menores en la atención abierta, permiten disminuir prestaciones en la atención cerrada, argumento que debe considerarse para asegurar medicamentos de calidad $\mathrm{y}$ en forma continua para estos pacientes. En nuestra serie la frecuencia de hospitalizaciones tendió a disminuir, pero esta reducción no llegó a ser estadísticamente significativa tal vez por falta de tiempo de observación o probablemente porque la mayoría de las hospitalizaciones están generadas por un grupo de muy difícil control que deberían analizarse separadamente.

Identificamos un grupo particular de asmáticos $(12,5 \%)$, de gran severidad y difícil control, en quienes a pesar que su tratamiento incluyó dosis altas de esteroides no lograron estabilizarse, lo que concuerda con estudios norteamericanos que comunican que el $10 \%$ de los pacientes concentra el $44 \%$ de los gastos por asma ${ }^{10}$. Estimamos que un porcentaje de ellos se controlaría mejor si pudieran acceder a medicamentos que usualmente no están en nuestros hospitales (Ej: $\beta 2$ acción prolongada, Fluticasona). El mejor manejo de este pequeño grupo de asmáticos que genera la gran mayoría de las consultas en urgencia y hospitalizaciones constituye un desafío para los servicios públicos.

\section{Agradecimientos}

Los autores agradecen al Dr. Sergio Bello S., por su generosa colaboración en el inicio de este programa.

\section{Bibliografía}

1.- BELLO S, ITURRA C, SEPÚLVEDA R. Aplicación práctica del manejo del asma de adultos en el nivel primario. Consenso Chileno para el diagnóstico y manejo del Asma Bronquial en adultos. Rev Chil Enf Respir 1995; 199-204.

2.- MORENO R, CRUZ E. Diagnóstico de asma en adulto. Consenso Chileno para el diagnóstico y manejo del Asma Bronquial en adultos. Rev Chil Enf Respir 1995; 11: 173-6.

3.- Consenso Nacional en Enfermedad Pulmonar Obstructiva Crónica. Capítulo I, nomenclatura y definiciones. Rev Chil Enf Respir 1998; 14: 69-70.

4.- MEDINA E, KAEMPFFER A. Características y manejo de la morbilidad en siete ciudades chilenas. Rev Méd Chile 1997; 125: 950-5.

5.- MURTAGH E, HEANEY L. Prevalence of obstructive lung disease in a general population sample: the NICECOPD study. Eur J Epidemiol 2005; 20: 443-53.

6.- Global strategy for asthma management and prevention. National Institutes of Health. Publication $\mathrm{N}^{\circ}$ 023659, National Heart, Lung and Blood Institute 2002.

7.- SERRA-BATLES J, PLAZA V, BRUGUES J. Estudio poblacional del asma en una comarca de Barcelona. Coste generado por la enfermedad. Archivos de Bronconeumología 1996; 32: 4ls.

8.- BARROS M, GUTIÉRREZ M, ROJAS A. Clasificación de severidad y tratamiento en la fase estable en adultos. Consenso Chileno para el diagnóstico y manejo del asma bronquial en niños y en adultos. Rev Chil Enf Respir 1995; 11: 177-82.

9.- RUTTEN-VAN MOLKEN M, VAN DOORSLAER E, JANSEN M, KERSTJENS H, RUTTEN F. Cost and effects of inhaled corticosteroids and bronchodilators in asthma and Chronic Obstructive Pulmonary Disease. Am J Respir Crit Care Med 1995; 151: 975-82.

10.- WHITEMAN I A, GADUZO S C. The management of mild-to-moderate asthmatics in general practice: a retrospective analysis. Br J Med Econ 1993; 6: 25-35.

\footnotetext{
Correspondencia a:

Dr. Mauricio Riquelme O.

Bellavista 123, of. 402

Fono y Fax: (65) 289409

E-mail: mauricioriquelme@entelchile.cl

Puerto Montt, Chile
} 